\title{
Can Regulatory Focus Help Explain Why We Shop Differently Online? - A Proposed Model and Research Agenda
}

\author{
Peter Atorough \\ The Robert Gordon University \\ E-Mail: p.t.atorough1@ @rgu.ac.uk \\ Bill Donaldson \\ The Robert Gordon University \\ E-Mail: w.g.donaldson@rgu.ac.uk
}

\begin{abstract}
As an important behavioral trait in consumer psychology, regulatory focus (RF) has been proven to describe and predict human behavior in numerous situations as it demonstrates that individuals can be distinguished according to two independent structures of strategic inclination and orientation in the pursuit of goals: promotion focus and prevention focus. However, to date no research has explicitly considered the potential link between RF and perceived risk in the various dimensions of online shopping (OS). This paper aims to fill this gap. By developing an RF model of online consumer shopping behavior (REFCOS) covering motives, behaviors, and continuance-evaluation and empirically testing a number of hypotheses, we find how consumers with different foci use the Internet. Our findings provide confirmatory evidence for emerging literature that RF is a powerful predictor of behavior in OS.
\end{abstract}

Keywords: Internet shopping, Regulatory Focus Theory, Consumer Behavior, REFCOS

\section{INTRODUCTION}

According to a recent Google-commissioned report (Kalapesi, Willersdorf, and Zwillenberg, 2010), Internet-related commerce in the UK accounts for as much as $7.2 \%$ of the GDP and represents the fastest growing area of the economy. Although Internet commerce has continued to witness exponential growth, the quantity of 
research focusing on its antecedents has only recently begun to reflect this trend; many firms remain unclear about the factors that shape consumers' behavior online (Jayawardhena, Wright, and Dennis, 2007; Constantinides, Lorenzo, and Gómez-Borja, 2010). Early theories of online consumer behavior applied generically technology-oriented frameworks like Davis et al.'s (1989) Technology Acceptance Model, the Innovation Diffusion Theory (Rodríguez-Ardura, Martínez-López, and Luna, 2009), and the Unified Theory of Acceptance and Use of Technology (Noort, Kerkhof, and Fennis, 2008) (for example applications, see (Chen, Chen, and Paul, 2001; $\mathrm{Ha}$ and Stoel, 2008)). These technology-based theories' use of consumer-centered psychological variables has acted to increase our understanding of the dynamic nature of consumers in e-business scenarios. We now know that cognitive and affective processes such as perceptions and attitudes significantly influence why and how consumers shop online as well as their evaluation of online shopping (OS) (Venkatesh, Morris, Davis, and Davis, 2003); we also know that the effects of these cognitive and affective features differ by consumer (Werth and Foerster, 2007; Dennis, Merrilees, Jayawardhena, and Wright, 2009). Hence, we can evaluate OS differences from the perspective of psychological trait theories (Jayawardhena, 2004; Carla and Carlos, 2009). Similar to other channels of shopping, online shoppers have been classified according to various characteristics (Brown, Pope, and Voges, 2003). Nevertheless, as Rodriguez-Ardure et al. (2009) point out, the increased use of the Web as a marketing and purchasing channel has caused a shift in the research focus to questions directly related to consumers and their behavior.

Although the level of incipient research thus far is appreciable, with respect to psycho-cognitive influences in OS, many important variables have yet to be clearly assessed (Jayawardhena et al., 2007), especially within a theoretical context (Forsythe and Shi, 2003). For example, it is surprising that-despite the well-established behavioral prediction trait in extant literature - one factor that has only recently been examined to determine its effect on online consumer behavior is a consumer's regulatory focus (RF) (see (Higgins, 1997; 1998; 2000; 2002; Higgins, Shah, and Friedman, 1997). The surprise arises because, as early as 1997, Higgins identified the effects of RF as an underlying self-regulatory trait on personality and behavior and proposed the Regulatory Focus Theory (RFT), with subsequent research (Brockner, Higgins, and Low, 2004; Bryant and Dunford, 2008) demonstrating that individuals can be distinguished according to two independent structures of strategic inclination and orientation in the pursuit of goals: the promotion focus (emphasizing the presence of positive outcomes while minimizing errors of omission) and the prevention focus 
(favoring the absence of negative outcomes and the minimization of errors of commission) (Haws, Dholakia, and Bearden, 2010). Considering that shopping, in general, and online shopping, in particular, are preoccupied with positive and/or negative outcomes, which can result from commission or omission, the said surprise becomes self-evident. A number of seminal studies have introduced the concept of RF to online shopping, including its potential influence on perceived risk (Tsai and Huang, 2009; Noort, 2009), but these studies adopted the view of RF as a situational variable, whereas the current research is interested in the alternative conceptualization of RF as an enduring personality trait (Ha and Stoel, 2008). Furthermore, previous studies addressed adoption motivation but did not fully evaluate the holistic effect of RF on online shopping by capturing key conative and affective dimensions (e.g., actual usage behavior and consumers' evaluation of OS attributes).

This lack of research explicitly focusing on RF and online consumer behavior represents a gap in our knowledge of the subject. Thus, the current paper addresses a number of important questions: (i) to what extent, if at all, does a consumer's RF affect his/her use of Internet shopping? Specifically, (ii) can a consumer's RF predict his/her motivation for OS adoption? (iii) Can RF predict a consumer's actual OS behavioral preferences? (iv) Can RF predict a consumer's assessment or evaluation of attributes of the OS medium? In an attempt to answer these questions, the current study develops a theoretical model of online consumer shopping behavior based upon the RF classifications of promotion and prevention focus (Higgins et al., 1997), which we term the regulatory focus classification of online shoppers (REFCOS). We build upon Cheung et al.'s (2003) model of intention, adoption, and continuance (MIAC) framework which suggests three key Internet research streams covering the processes of (i) adoption and usage motivations (motives), (ii) actual adoption and usage (behaviors), and (iii) post-adoption usage and attribute assessment (continuance-evaluation) (see also (Brown et al., 2003; Carla and Carlos, 2009; Chen et al. 2001)). We begin with an overview of RFT, based upon which we propose and describe our REFCOS framework; we then proceed to express a number of propositions that help establish the research agenda.

In general, knowing why and how different consumer segments use the Internet and which attributes they value most may provide researchers and practitioners with valuable information about which factors inform consumer choices online. Consistent with this reasoning, the REFCOS model may prove to be a useful tool in targeting and predicting online consumers by knowing their regulatory focus; for example, REFCOS can provide a new perspective for understanding differences in consumers' 
online risk perception, avoidance, loyalty, and dependency (Chen, Tarn, and Han, 2004; Taylor, Bagby, and Parker, 1997).

\section{REGULATORY FOCUS THEORY}

Higgins' (1997) theory of regulatory focus states that different psychological systems exist in individuals that have a direct effect on how the individuals approach goals and objectives. Some individuals have a higher need for attainment of positive outcomes, thereby directing their attention to the maximization of gains, whereas others have a higher need for protection against the occurrence of unpleasant states and the avoidance of negative consequences, thereby directing their attention to the minimization of losses. To illustrate, an individual who is "promotion focused" would, according to RFT, be more receptive to positively framed (gains/non-gains) messages rather than negatively framed (losses/non-losses) ones; on the other hand, an individual who is "prevention focused" would be more affected by negatively framed messages than positively framed ones. This effect would extend to consumer behavior situations, where a promotion-focused person's decision to purchase would be heavily influenced by hedonic attributes of the object as opposed to a prevention-focused person's predominant consideration of the performance and reliability of the object (the utilitarian objective) (Vellido, Lisboa, and Meehan, 2000).

RF can represent an enduring personality feature - namely, the dispositional or chronic view of RF (Higgins, 1997; 1998). However, RF can also be determined by the situation; it may be influenced by the environment, the decision-making process, or the magnitude of the consequences of the decision to be made (Forster, Higgins, and Bianco, 2003; Forster, Higgins, and Idson 1998). Although RFT assumes that all individuals can be classified as chronically belonging to one focus or the other, it is not clear to what extent situational-induced RF affects preexisting dispositions: Does the situation simply reinforce the chronic trait or are situational influences strong enough to alter the enduring focus completely? For example, does online shopping, by its acknowledged uniquely risky nature (Noort, 2009), induce a prevention focus irrespective of shoppers' natural predispositions? Or does online shopping, due to its very nature, reinforce promotion focus or prevention focus depending on the consumer's chronic disposition? In this research, we are primarily interested in RF effects of the chronic type.

Irrespective of whether chronic or situational induced, an individual's RF orientation at any given time has consequences for key behavioral determinants, such as information processing, motivation, and decision making (Vellido et al., 2000), 
thereby influencing which aspects of a message or presentation an individual specifically seeks out or pays attention to and retains. Based on this reasoning, we contend that RF in its various manifestations plays an influential role in OS consumers' perception of and attention to risk as well as response to marketing content.

\section{REGULATORY FOCUS AND ONLINE SHOPPING: A PROPOSED MODEL}

Rodriguez-Ardure et al. (2009) proposed that current research into behavioral attributes of online shopping could be structured into four lots: study of purchase intention variables, analysis of the actual purchasing process, satisfaction and Web loyalty, and the adoption of models/theories to the electronic market. Similarly, Cheung et al. (2003) proposed MIAC as an integrated base model. The MIAC models the antecedent factors that affect intention, adoption, and continuance, but it does not specifically model behavioral outcomes or the inputs of individual psychological traits. However, it has been suggested that the literature on OS behavior can be structured along a pattern of cognitive outcomes that follow from consumers' motivation for shopping online in the first place to how they behave in this context and their evaluation (or perception) of the OS attributes (Chiang and Dholakia, 2003; Forsythe and Shi, 2003; Vijayasarathy, 2004). Therefore, it is reasonable to propose a model that simultaneously depicts consumer differences on the key dimensions of motivation, behavior, and evaluation, thereby providing a holistic view of online cognitive and conative outcomes for the three stages of the OS process. Given its antecedents, we contend that RF provides a sound basis for achieving such a model.

We assert that psycho-cognitive factors of consumer differences in motivations for shopping online, actual online behavior, and evaluation or perception of attributes can be broadly grouped into bipolar classes along a regulatory focus perspective. Guided by previous research on RFT and the body of work on OS motivations, usage, and evaluation, we propose the regulatory focus classification of online shoppers (REFCOS) model (Figure 1). 

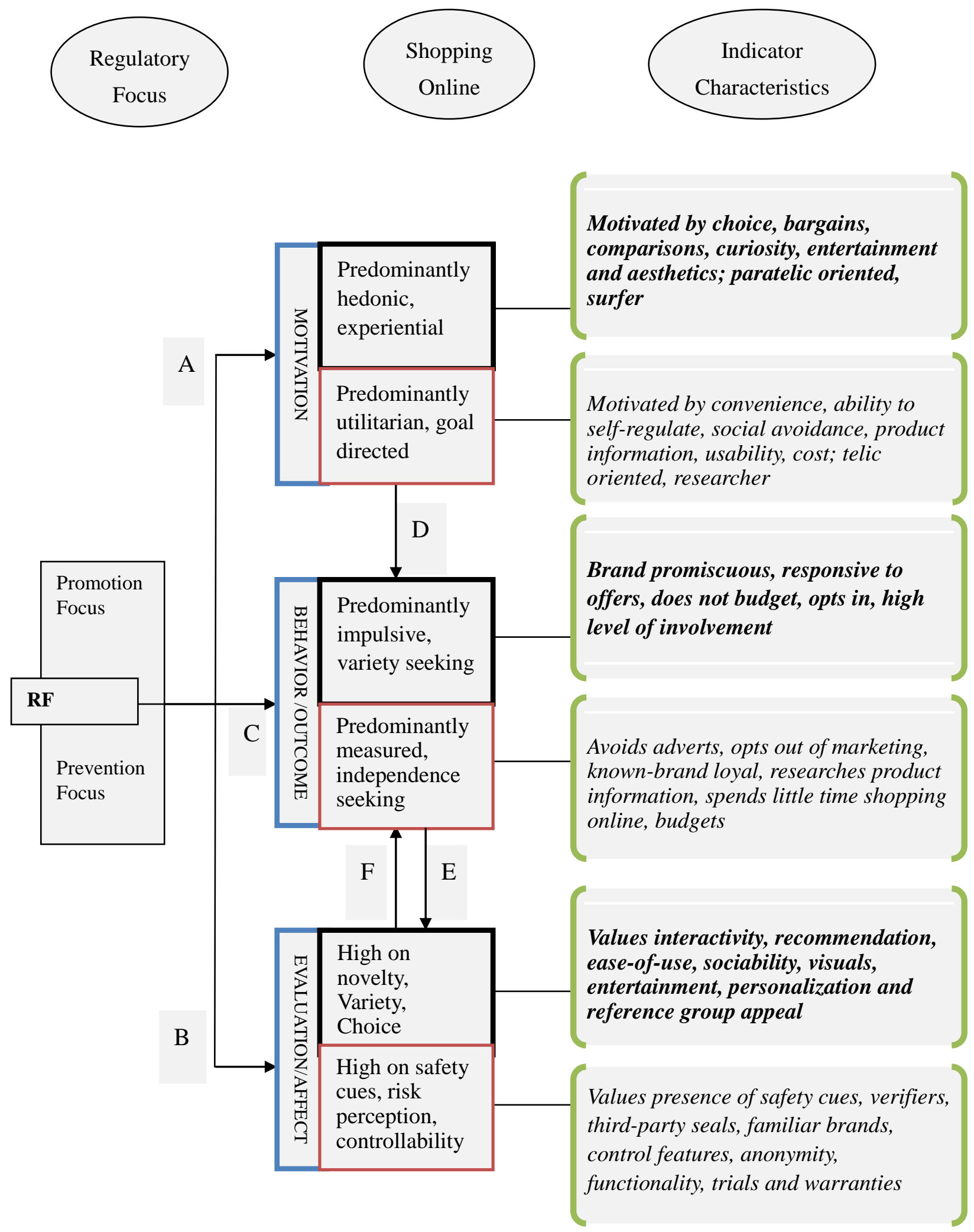

Figure 1 Regulatory focus classification of online shoppers (REFCOS) model 
The REFCOS framework combines the functional, behavioral, and cognitive paradigms in a structural manner and addresses specific questions: Does the motivation for and orientation to online shopping differ according to consumers' RF (A)? Does regulatory focus affect the actual behavior of consumers online (C), and does it influence the evaluation of OS attributes (B)? Consistent with extant literature, other relationships proposed suggest that the process of shopping online leads from motive to usage (D) and from usage to evaluation of outcomes (E). However, evaluation also precedes usage outcomes both before (expectation) and after (continuance) (F) (see also (Moran, 1999)).

Consequently, REFCOS can be summarized by a number of propositions:

Proposition 1a: Consumers' motivation for adopting Internet shopping is related to their regulatory focus.

Proposition 1b: Prevention-focused consumers will be predominantly motivated by utilitarian objectives; conversely, promotion-focused consumers will be predominantly motivated by hedonic objectives.

Proposition 2a: Consumers' usage behavior in Internet shopping is related to their regulatory focus.

Proposition 2b: Prevention-focused consumers will exhibit predominantly control-seeking behaviors; conversely, promotion-focused consumers will exhibit predominantly fun-seeking behaviors.

Proposition 3a: Consumers' affect for Internet shopping medium attributes is related to their regulatory focus.

Proposition 3b: Prevention-focused consumers will have a higher positive affect toward safety-oriented attributes than toward variety-oriented attributes; conversely, promotion-focused consumers will have a higher positive affect toward variety-oriented attributes than toward safety-oriented attributes.

\section{DISCUSSION AND IMPLICATIONS}

Although applied and tested within several important areas of human behavior, regulatory focus has remained a scantly explored area in consumer behavior. Recent research output in this area is indicative of renewed activity and interest, especially from a consumer behavior point of view; this recent research is particularly illuminative of the effect of RF on consumer behavior, especially in the realm of online shopping behavior. Our research aims to further stimulate debate on this topic of significant impact on marketers, given that current OS growth is unprecedented; 
thus, understanding factors (demographic, psychological, and otherwise) that affect behavioral outcomes in this channel of shopping can only aid in the quest for better results and best practices. The model proposed in this paper and the development of our propositions set a relevant research agenda for further evaluation. The next stage would be to select an appropriate sample of online shopping consumers to empirically evaluate the propositions put forward in this paper. Given adequate responses, the findings from this study will be important to both academia and practice. They will be particularly relevant to retailers and marketers, who will be interested in knowing the psycho-dynamics of their consumers and potential market segments.

In conclusion, the evidence provided from extant literature on regulatory focus theory and its application to several dimensions of human behavioral variables, as well as its recent assessment in the relationship between consumers and Internet shopping, appears to strongly support a holistic model of consumer behavior online based on a better illustration of regulatory focus effects in this shopping medium. The REFCOS model aims to serve this very purpose.

\section{REFERENCES}

Brockner, J., Higgins, E. T., and Low, M. B. (2004). Regulatory focus theory and the entrepreneurial process. Journal of Business Venturing, 19(2), 203-207.

Brown, M., Pope, N., and Voges, K. (2003). "Buying or browsing?" An exploration on shopping orientations and online purchase intention. European Journal of Marketing, 37(11/12), 1666-1684.

Bryant, P., and Dunford, R. (2008). The influence of regulatory focus on risky decision making. Applied Psychology, 57(2), 335-359.

Carla, R. M., and Carlos, L. N. (2009). Segmenting consumers by e-shopping behaviour and online purchase intention. Journal of International Business. Retrieved October 21, 2009, from http://jib.debii.curtin.edu.au/iss03_mafe.pdf

Chen, K., Chen, I., and Paul, H. (2001). Explaining online behavioral differences: an Internet dependency perspective. The Journal of Computer Information Systems, 41(3), 59-63.

Chen, K., Tarn, M., and Han, B. T. (2004). Internet dependency: Its impact on online behavioral patterns in e-commerce. Human Systems Management, 23(1), 49-58. 
Cheung, C. M. K., Zhu, L., Kwong, T., Chan, G. W., and Limayem, M. (2003). Online consumer behaviour: a review and agenda for future research. Proceedings of the 16th Bled E-Commerce Conference, Bled, 9-11 June, 2003. Retrieved September 12, 2009, from http://ecom.fov.unimb.si/proceedings.nsf/0/c9beda3ca328c8b7c1256ea1002c72b 8/\$FILE/16Cheung.pdf

Chiang, K. P., and Dholakia, R. R. (2003). Factors driving consumer intention to shop online, Journal of Consumer Psychology, 13(1), 177-183.

Constantinides, E., Lorenzo, C., and Gómez-Borja, M. A. (2010). Effects of Web experience on consumer choice: a multicultural approach. Journal of Internet Research, 20(2), 188-209.

Davis, F. D., Bagozzi, R. P., and Warshaw, P. R. (1989). User acceptance of computer technology: a comparison of two theoretical models. Management Science, 35(8), 982-1003.

Dennis, C., Merrilees, B., Jayawardhena, C., and Wright, L. T. (2009). E-consumer behaviour. European Journal of Marketing, 43(9/10), 1121-1139.

Forster, J., Higgins, E. T., and Bianco, A. T. (2003). Speed/accuracy in performance: Tradeoff in decision making or separate strategic concerns? Organizational Behavior and Human Decision Processes, 90(1), 148-164.

Forster, J., Higgins, E. T., and Idson, L. C. (1998). Approach and avoidance strength during goal attainment: Regulatory focus and the "goal looms larger" effect. Journal of Personality and Social Psychology, 75(5), 1115-1131.

Forsythe, S. M., and Shi, B. (2003). Consumer patronage and risk perceptions in Internet shopping. Journal of Business Research, 56(11), 867-875.

Ha, S., and Stoel, L. (2008). Promoting customer-retailer relationship building: influence of customer trustworthiness of customer loyalty programme marketing. Journal of Customer Behaviour, 7(3), 215-229.

Haws, K. L., Dholakia, U. M., and Bearden, W. O. (2010). An assessment of chronic regulatory focus measures. Journal of Marketing Research, 47(5), 967-982.

Higgins, E. T. (1997). Beyond pleasure and pain. American Psychologist, 52(12), 1280-1300.

Higgins, E. T. (1998). Promotion and prevention: regulatory focus as a motivational principle. Advances in Experimental Social Psychology, 30(1), 1-46.

Higgins, E. T. (2000). Does personality provide unique explanations for behaviour? Personality, a cross-person variability in general principles. European Journal of Personality, 14(5), 391-406. 
Higgins, E. T. (2002). How Self-Regulation creates distinct values: the case of Promotion and Prevention decision making. Journal of Consumer Psychology, 12(3), 177-191.

Higgins, E. T., Shah, J., and Friedman, R. (1997). Emotional responses to goal attainment: strength of regulatory focus as moderator. Journal of personality and social psychology, 72(3), 515-525.

Jayawardhena, C. (2004). Personal values' influence on e-shopping attitude and behaviour. Internet Research, 14(2), 127-138.

Jayawardhena, C., Wright, L. T., and Dennis, C. (2007). Consumers online: intentions, orientations and segmentation. International Journal of Retail \& Distribution Management, 35(6), 515-525.

Kalapesi, C., Willersdorf, S., and Zwillenberg, P. (2010). The Connected Kingdom: how the Internet is transforming the UK economy. Boston: BCG.

Moran, N. (1999). Brand and trust on the Internet: how to build Amazon.com. Chemical Week, 18/08/1999, S14-S15.

Noort, G. van. (2009). Validating the persuasiveness of online safety cues: The effect of multiple cues on online spending. Proceedings of the 8th ICORIA Conference, Klagenfurt, 8, (Unnumbered electronic version).

Noort, G. van, Kerkhof, P., and Fennis, B. M. (2008). The persuasiveness of online safety cues: the impact of prevention focus compatibility of web content on consumers' risk perceptions, attitudes, and intentions. Journal of Interactive Marketing, 22(4), 58-72.

Rodríguez-Ardura, I. J., Martínez-López, F., and Luna, P. (2009). The consumer and the Web: a critical revision of the contributions to Web science from the marketing and the consumer behaviour discipline. Proceedings of the WebSci'09: Society On-Line, Athens, 18-20 March 2009. Retrieved December 21, 2010, from http://journal.webscience.org/135/

Taylor, G., Bagby, R., and Parker, J. (1997). Disorders of affect regulation. Cambridge: Cambridge University Press.

Tsai, H., and Huang, H. (2009). Online consumer loyalty: why e-tailers should seek a high-profile leadership position. Computers in Human Behavior, 25(6), 1231-1240.

Vellido, A., Lisboa, P. J., and Meehan, K. (2000). Quantitative characterization and prediction of on-line purchasing behavior: a latent variable approach. International Journal of Electronic Commerce, 4(4), 83-104. 
Venkatesh, V., Morris, M. G., Davis, G. B., and Davis, F. D. (2003). User acceptance of information technology: Toward a unified view. MIS Quarterly, 27(3), 425-478.

Vijayasarathy, L. R. (2004). Shopping orientations, product types and internet shopping intentions. Electronic Markets, 13(1), 67-79.

Werth, L., and Foerster, J. (2007). How regulatory focus influences consumer behavior. European Journal of Social Psychology, 37(1), 33-51. 
